\title{
Performance optimization of an oil ellipse gerotor pump for automotive engine
}

\author{
${ }^{*}$ Xiaohu Sang ${ }^{1 \mathrm{a}}$, Xiaojun Zhou ${ }^{1 \mathrm{~b}}$, Xiaoguang Liu ${ }^{1 \mathrm{c}}$ \\ ${ }^{1}$ School of Mechatronics Engineering and Automation,Shanghai University,Shanghai,China \\ ”asangxh7@qq.com, bsdzhouxj@shu.edu.cn, 'xiaoguangvisa@126.com
}

\begin{abstract}
Keywords: Ellipse gerotor pump; Engaging limiting point; Optimization design; Bench test
Abstract. The internal cycloidal gear pump is one kind of oil pump, which consists of pump body, pump cover and rotors. It is widely used in the range of vehicles. A gerotor pump has various advantages such as compact size lower noise, less emissions and little pressure ripples for circulation of the fluid. In practice, the conventional cycloid rotor oil pump has the problem of exists engaging limit point which results in bad wear of rotors, reduce the volumetric efficiency. For above these problems, we use the higher efficient transmission performance ellipse rotors. After that, we propose a new method of searching a new point in original ellipse profile to remove the engaging limit point. Experimental investigation has been carried out through bench test, and the results show that at the range of $0.5 \mathrm{MPa}$ to $2.5 \mathrm{MPa}, 3 \%$ and $5 \%$ increase of the volumetric efficiency and volumetric flux of the gerotor pump has been achieved respectively through the optimization of new rotors profile. It explains this method of rotor profile by removing engaging limiting point satisfies he design requirements without any volume size changing.
\end{abstract}

\section{Introduction}

Gerotor pump is a critical component in engine lubrication oil system, it keeps the whole system working in a good situation. Its characteristics of pressure, rev and flux will have great effect on the basic performance of engine directly. Generally, the oil pump is made up of oil chamber, inner and outer rotor, groove, inlet and outlet. The outer-rotor has one more tooth than the inner rotor ${ }^{[1]}$. Fig. 1 and Fig. 2 show the wear of inner and outer rotor.

Some important literatures on oil pump can be found: For example, Colbourne ${ }^{[2]}$ proposed a geometry method to find the envelopes of trochoids that perform a planetary motion. Demenego et al. $^{[3]}$ developed a tooth contact analysis (TCA) computer program and discussed avoidance of tooth interference and rapid wearing through modification of the rotor profile geometry of a cycloidal pump whose one pair of teeth is in mesh at every instant. Mimmi and Pennacchi ${ }^{[4]}$ obtained $^{2}$ transcendental equations for the calculation of the limit dimensions to avoid undercutting. Hwang and $\mathrm{Hsieh}^{[5]}$ presented a geometry design procedure based upon the theories of envelope and conjugate surfaces for the hypotrochoidal gear pump using the equidistant extended hypotrochoid curve.

However, most earlier studies focused on the commercially available oil pump using the equidistant shortened epitrochoid curve to the authors' best knowledge. This paper presents the method on rotors profile optimization of eliminate the engaging limiting point. we search a new point $F$ in in original ellipse profile and replace it to a pair of conjugate circle, the radius can be achieved by the relationship. Next, the experimental investigation has been carried out through bench test. Compared with the origin profile rotor oil pump, the new optimized pump has higher volumetric efficiency and volumetric flux. 


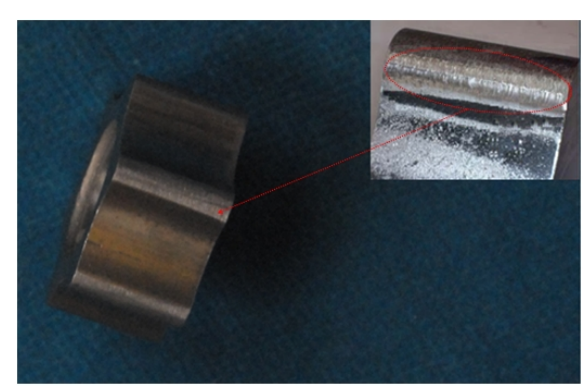

Fig.1 wear of inner rotor

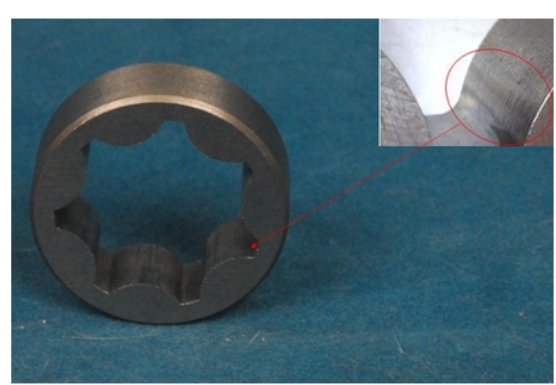

Fig. 2 wear of outer rotor

\section{Profile Optimization}

Fig. 3 shows the scheme of ellipse rotor profile, outer rotor profile is ellipse. In the Fig.5, point $C$ is the rotors crest of the original curves, point $A$ is the rotors tooth dedendum. The arc $A B$ is the engaging profile, point $B$ is the engaging limit point. Arc $D C$ is the circular arc; while the arc $B D C$ belongs to the separating zone, the rotors are do not engaging here. In order to make the pump keeps a continuously transmission, the engaging limit point $B$ must be removed, and the arc $B D C$ should be in engaging too. Besides, the pump will gain an additional conjugated sealing.

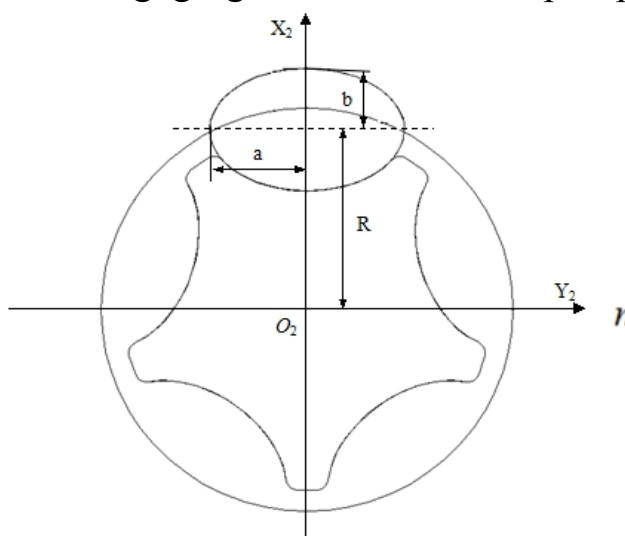

Fig.3. Scheme of ellipse oil pump

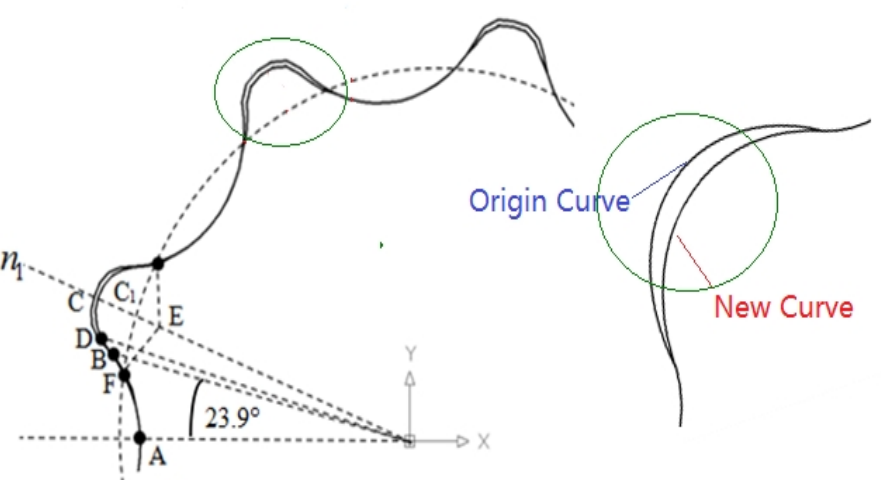

Fig.4. Design result of new pump curve

In the article, a new method has been proposed, that is redesign a new ellipse curve between the rotors crest point $A$ and rotors tooth dedendum point $B$. Choose a random point $F$ on the ellipse curve $A B$, let the point $F$ to be the contact point of the ellipse circle curve. Therefore, the Length between point $B$ and point $F$ is $B F$, suppose the $B F=\Delta S$, while, $\Delta S=A B-A F$.

According to the $A F$ ellipse Eq.(1) and Eq.(2):

$$
\begin{aligned}
& \left\{\begin{array}{l}
x_{1}=a \sin \theta \\
y_{1}=R-b \cos \theta
\end{array}\right. \\
& \Delta s=\int_{\theta_{f}}^{\theta_{c}} \sqrt{a^{2} \cos ^{2}(\theta)+b^{2} \sin ^{2}(\theta)} d \theta
\end{aligned}
$$

we find that when the value of $\Delta S$ become more the rotors, the friction will decreased. Besides, coordinate point of point $\mathrm{F}$ can be expressed as Eq.(3):

$$
\left\{\begin{array}{l}
x_{F}=a \sin \theta \\
y_{F}=R-b \cos \theta
\end{array}\right.
$$

The outer-rotor teeth number is 9 , according the equal tooth number principle, the degree of one tooth is 360/9, drawing a symmetric line $Z$ via the geometrical center of outer-rotor $\mathrm{O}_{2}$, then drawing the normal line of the outer-rotor via the point $F$ and intersect in symmetric line $Z$ with point $E$, let its coordinate to be $\left(X_{E}, Y_{E}\right)$, as shown in Eq.(4). 


$$
x_{E}=\frac{R+\frac{\left(a^{2}-b^{2}\right)}{b} \cos \left(\theta_{f}\right)}{\tan \left[\frac{\pi}{2}\left(\frac{z_{1}-1}{z_{1}+1}\right)\right]+\frac{a}{b} \cot \left(\theta_{f}\right)} \quad y_{E}=\frac{R+\frac{\left(a^{2}-b^{2}\right)}{b} \cos \left(\theta_{f}\right)}{\tan \left[\frac{\pi}{2}\left(\frac{z_{1}-1}{z_{1}+1}\right)\right]+\frac{a}{b} \cot \left(\theta_{f}\right)} \cdot \tan \left[\frac{\pi}{2}\left(\frac{z_{1}-1}{z_{1}+1}\right)\right]
$$

The minimum gap between the rotors is shown in Eq.(5) :

$$
\delta_{\min }=R-b+e-\sqrt{\left(x_{F}-x_{E}\right)^{2}+\left(y_{F}-y_{E}\right)^{2}}-\sqrt{x_{E}^{2}+y_{E}^{2}}
$$

Substituting the relation Eq.(3), (4) into Eq.(5) can get the value of the minimum gap, $\delta$. $\min =0.1 \mathrm{~mm}$.

From equation (1) to (5), we can confirm the more details of optimized profile parameters in Tab.1.

Tab.1. Optimized profile parameters

\begin{tabular}{|l|l|l|l|}
\hline Parameter & Value & & \\
\hline Arc length $\Delta s(\mathrm{~mm})$ & 3.2 & Coordinate $E$ & $(8.36,7.11)$ \\
\hline Small gap $\delta \min (\mathrm{mm})$ & 0.1 & Coordinate $F$ & $(10.89,5.03)$ \\
\hline Engaging point coordinate $B$ & $(11.02,5.22)$ & $\operatorname{Sin} \theta_{f}$ Cos $\theta_{f}$ & $0.94 ; 0.33$ \\
\hline
\end{tabular}

According to the result of the optimization, the final optimized rotors profile model can be obtained as shown in Fig5.

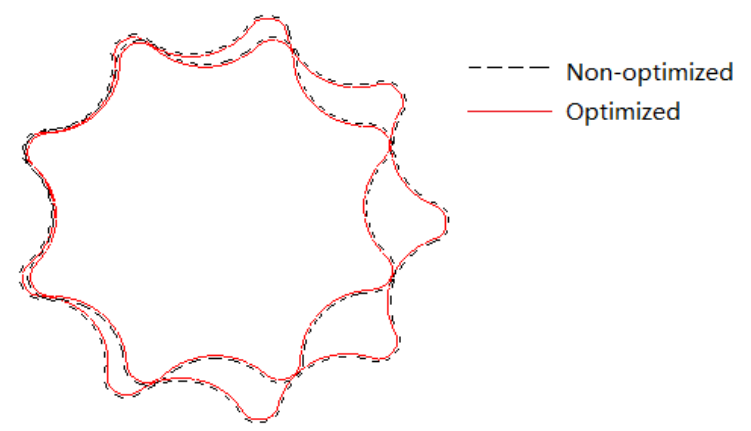

Fig.5. Non-optimized and optimized profile results

\section{Experiment}

Fig. 6 shows the whole experiment system. The oil is absorbed and flows into testing oil pump by the adjustable speed electric drive motor, a part of the oil flows into the thermostatic apparatus via the relief valve, the other part of the oil via the conditioning valve and directional control valve return into the thermostatic apparatus. The torque-rotating speed measure instrument is installed between the adjustable speed electric drive motor and testing oil pump; The humidity and pressure gage are fixed near the outlet of pump to monitor oil temperature and pressure; the vacuum pressure gauge is fixed on the inlet. Use the shell 10W-30 lubricant in test, keep the oil temperature about $60 \pm 5^{\circ} \mathrm{C}$. The pump inlet and outlet pressure are measured by the high accuracy pressure transmitter with $0.5 \%$ measurement error. The flow rate is measured by an electromagnetic flow meter with $0.3 \%$ measurement error. The motor rotating speed is measured by an infrared velocimeter with $\pm 0.1 \%$ maximum uncertainty. the vacuum pressure gauge is less level 0.5 . The oil temperature is measured by a temperature sensor with $\pm 0.5 \%$. In the end, flow field analysis is collected by the system and accomplished using TECPLOT software. 


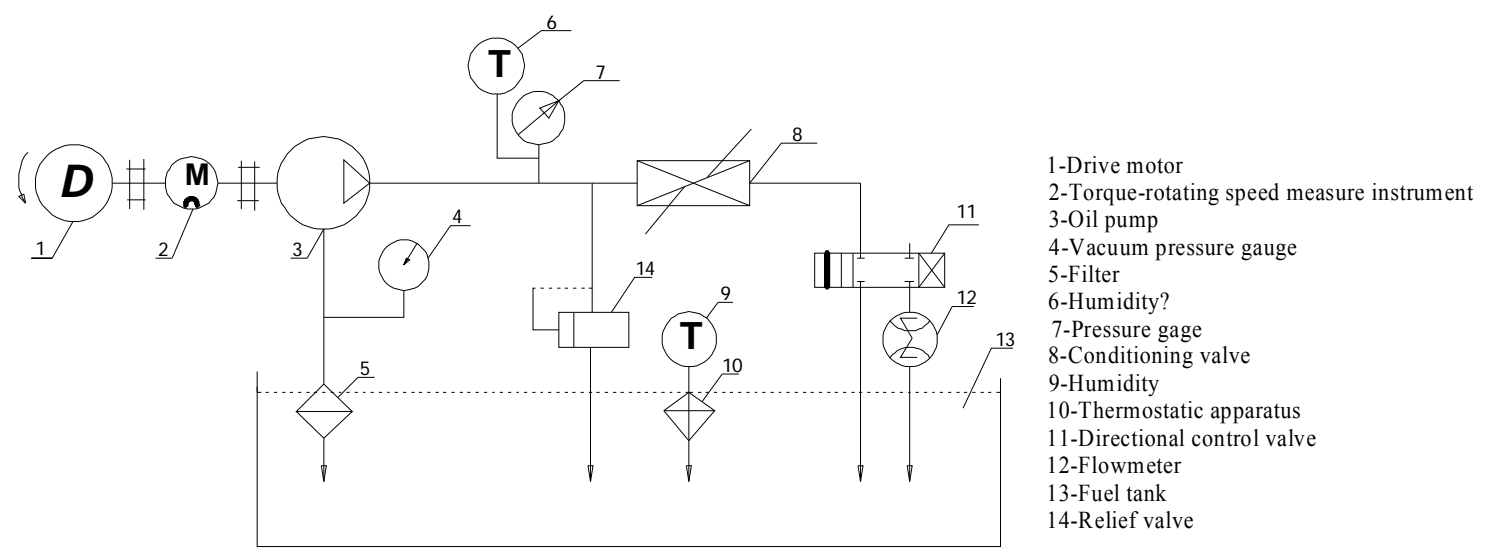

Fig.6. Experiment System

\section{Results}

In the experiment, the change of oil pressure is achieved by adjusting the valve opening. Fig.7 shows the volumetric flux at different pressure of the non-optimized and optimized profile, from the result, we can obtain: With the increasing of the oil pressure, the flux of the outlet get decreased, its main reason is the increased pressure bring leakage, the leakage lead to the low flux, while the optimized pump's flow curve is better than the non-optimized one at the range of $0.5 \mathrm{MPa}$ to $2.5 \mathrm{MPa}$. Fig. 8 shows the volumetric efficiency (VE) at different pressure of the non-optimized and optimized profile. Fig.9 shows the volumetric efficiency and fuel delivery of the non-optimized and optimized profile from $1000 \mathrm{r} / \mathrm{min}$ to $7000 \mathrm{r} / \mathrm{min}$.

Compared with the non-optimized pump, the results show that at the range of $0.5 \mathrm{MPa}$ to $2.5 \mathrm{MPa}$, $3 \%$ and $5 \%$ increase of the volumetric efficiency and volumetric flux of the gerotor pump has been achieved respectively. It explains this method of rotor profile by removing engaging limiting point satisfies he design requirements without any volume size changing.

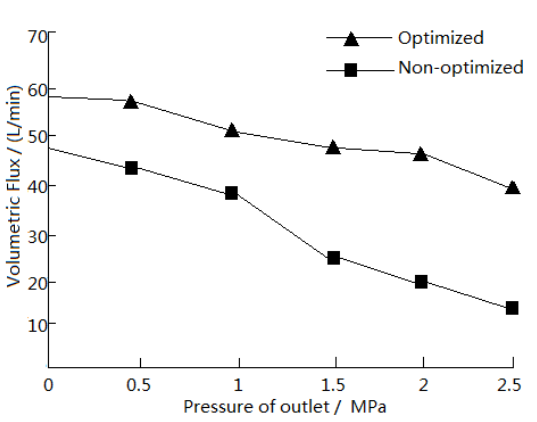

Fig.7.Flux characteristic curve

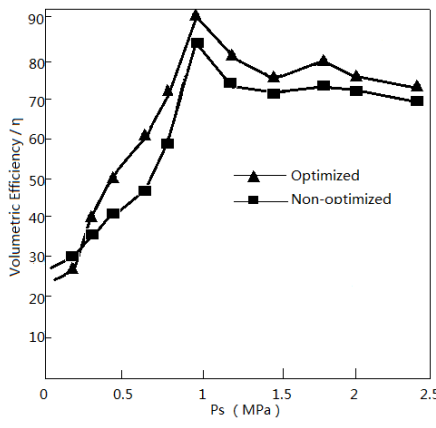

Fig.8.VE curve

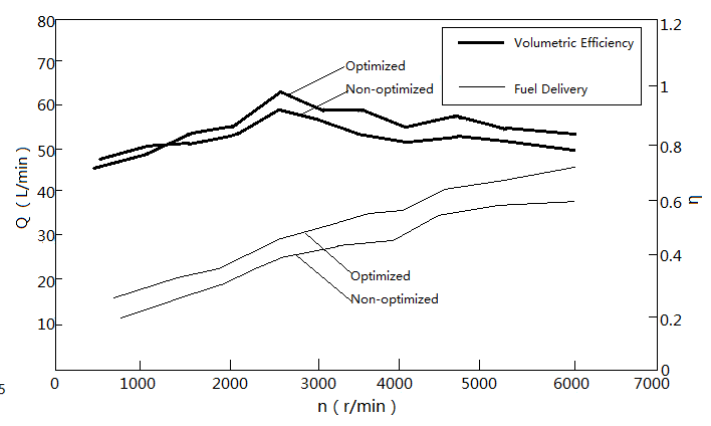

Fig.9.VE and fuel delivery curve

\section{Conclusions}

(1) The method of removing engaging limiting point is presented in the article, the minimum gap between the rotors $\delta \mathrm{min}$ is also obtained.

(2) Experimental investigation has been carried out through bench test, and the results show that When the oil pressure ranges from 0.5 to $2.5 \mathrm{Mpa}, 3 \%$ and $5 \%$ increase of the volumetric efficiency and volumetric flux of the gerotor pump has been achieved respectively through the optimization of new rotors profile.

(3) It explains this method of rotor profile by removing engaging limiting point satisfies he design requirements without any volume size changing. 


\section{References}

[1] Huang, J.X., Research for double-enveloping cycloid internal gearpump, Machine Tool \& Hydraulics. 4 (2010) 22-26.

[2] Colbourne, J.R., The geometry of trochoid envelopes and their application in rotary pumps, Mechanism and Machine Theory. 4 (1974) 421-435.

[3] Demenego, A., Vecchiato, D., Litvin, F.L., Nervegna, N., Manco, S., Design and simulation of meshing of a cycloidal pump, Mechanism and Machine Theory. (2002) 37, 311-332.

[4] Mimmi, G.C., \& Pennacchi, P.E., Non-undercutting conditions in internal gears, Mechanism and Machine Theory. 35 (2000) 477-490.

[5]Y.-W. Hwang and C.-F. Hsieh, Geometry design using hypotrichoid and non-undercutting conditions for an internal cycloidal gear, Transactions of the ASME, Journal of Mechanical Design, 129 (2007) 413-420 